University of Wollongong

Research Online

SMART Infrastructure Facility - Papers

Faculty of Engineering and Information

Sciences

$1-1-2020$

\title{
Fabrication of Aligned Biomimetic Gellan Gum-Chitosan Microstructures through 3D Printed Microfluidic Channels and Multiple In Situ Cross-Linking Mechanisms
}

Thomas Robinson

University of Wollongong, tmr463@uowmail.edu.au

Sepehr Talebian

University of Wollongong, st432@uowmail.edu.au

Javad Foroughi

University of Wollongong, foroughi@uow.edu.au

Zhilian Yue

University of Wollongong, zyue@uow.edu.au

Cormac Fay

University of Wollongong, cfay@uow.edu.au

See next page for additional authors

Follow this and additional works at: https://ro.uow.edu.au/smartpapers

Part of the Engineering Commons, and the Physical Sciences and Mathematics Commons

Research Online is the open access institutional repository for the University of Wollongong. For further information contact the UOW Library: research-pubs@uow.edu.au 


\title{
Fabrication of Aligned Biomimetic Gellan Gum-Chitosan Microstructures through 3D Printed Microfluidic Channels and Multiple In Situ Cross-Linking Mechanisms
}

\author{
Abstract \\ In this study we use a combination of ionic- and photo-cross-linking to develop a fabrication method for \\ producing biocompatible microstructures using a methacrylated gellan gum (a polyanion) and chitosan (a \\ polycation) in addition to lithium phenyl-2,4,6-trimethylbenzoylphosphinate (LAP) as the photoinitiator. \\ This work involves the development of a low-cost, portable 3D bioprinter and a customized extrusion \\ mechanism for controlled introduction of the materials through a 3D printed microfluidic nozzle, before \\ being cross-linked in situ to form robust microstructure bundles. The formed microstructures yielded a \\ diameter of less than $1 \mu \mathrm{m}$ and a tensile strength range of $\sim 1 \mathrm{MPa}$. This study is the first to explore and \\ achieve GGMA:CHT microstructure fabrication by means of controlled in-line compaction and photo- \\ cross-linking through 3D printed microfluidic channels.

\section{Keywords} \\ gellan, biomimetic, microfluidic, mechanisms, fabrication, cross-linking, aligned, situ, gum-chitosan, \\ microstructures, 3d, printed, channels, multiple

\section{Disciplines} \\ Engineering | Physical Sciences and Mathematics

\section{Publication Details} \\ Robinson, T. M., Talebian, S., Foroughi, J., Yue, Z., Fay, C. D. \& Wallace, G. G. (2020). Fabrication of Aligned \\ Biomimetic Gellan Gum-Chitosan Microstructures through 3D Printed Microfluidic Channels and Multiple \\ In Situ Cross-Linking Mechanisms. ACS Biomaterials Science and Engineering, Online First A-K.
}

\section{Authors}

Thomas Robinson, Sepehr Talebian, Javad Foroughi, Zhilian Yue, Cormac Fay, and Gordon G. Wallace 


\title{
Fabrication of Aligned Biomimetic Gellan Gum-Chitosan Microstructures Through 3D Printed Microfluidic Channels and Multiple In-Situ Crosslinking Mechanisms
}

\author{
Thomas M. Robinson ${ }^{1}$, Sepehr Talebian ${ }^{1,2}$, Javad Foroughi ${ }^{1,4}$, Zhilian Yue ${ }^{1}$, Cormac D. Fay ${ }^{1,3}$ and Gordon G. Wallace ${ }^{1 *}$ \\ ${ }^{1}$ Intelligent Polymer Research Institute (IPRI), ARC Centre of Excellence for Electromaterials Science (ACES), \\ Australian Institute for Innovative Materials (AIIM), University of Wollongong, Innovation Campus, \\ Wollongong, NSW 2522, Australia. \\ ${ }^{2}$ Illawarra Health and Medical Research Institute (IHMRI), University of Wollongong, Wollongong, NSW, 2522 \\ Australia. \\ ${ }^{3}$ SMART Infrastructure Facility, Engineering and Information Sciences, University of Wollongong, NSW 2522, \\ Australia. \\ ${ }^{4}$ School of Electrical, Computer and Telecommunications Engineering, Faculty of Engineering and Information \\ Sciences, University of Wollongong, NSW 2522, Australia.
}

* Corresponding author: gordon_wallace@uow.edu.au

\begin{abstract}
In this study we use a combination of ionic- and photo- crosslinking to develop a fabrication method for producing biocompatible microstructures using: a methacrylated gellan gum (a polyanion) and chitosan (a polycation) in addition to Lithium phenyl-2,4,6-trimethylbenzoylphosphinate (LAP) as the photo-initiator. This work involves the development of a low-cost, portable 3D Bioprinter and a customised extrusion mechanism for controlled introduction of the materials through a $3 \mathrm{D}$ printed microfluidic nozzle, before being cross-linked in situ to form robust microstructure bundles. The formed microstructures yielded a diameter of less than $1 \mu \mathrm{m}$ and a tensile strength range of $\sim 1 \mathrm{MPa}$. This study is the first to explore and achieve GGMA:CHT microstructure fabrication by means of controlled in-line compaction and photo-crosslinking through 3D printed microfluidic channels.
\end{abstract}

Keywords: 3D Printing, Hydrogel, Micro, Structures, Microfluidics, Microfabrication, Gellan gum, Chitosan, Biomimetic Collagen, Biomimicry, In-situ, UV Crosslinking, Photo Crosslinking, LAP. 


\section{Introduction}

The use of 3D printing to create ordered structures comprised of biomaterials is well established ${ }^{1}$, with efforts often exploring three key aspects: materials for biocompatibility and printability ${ }^{2-4}$, cell growth and differentiation ${ }^{5,6}$, and fabrication techniques ${ }^{7,8}$. Most studies have focused on a single material and the use of extrusion printing to form a desired construct. Other approaches allow the introduction of multiple materials. For example, coaxial printing was established to allow for the fabrication of constructs with more complicated architectures, befitting certain tissue engineering applications such as generation of vascularized tissues ${ }^{9,10}$. In a similar vein, more complicated 3D printing platforms were designed to allow for the fabrication of functional bionic organs with anatomic geometries, such an ear or heart ${ }^{11,12}$. Interestingly, these ad-hoc $3 \mathrm{D}$ printing platforms can be applied to different fabrication modalities, such as electrospinning ${ }^{13}$ or melt electro-writing ${ }^{14}$, to fabricate structures with nanometric elements and/or structure-hydrogel composites ${ }^{15,16}$.

The ability to introduce multiple materials provides a route to engineering the appropriate combination of mechanical and bioactive properties ${ }^{17,18}$. A broad range of biopolymers have been examined to institute 3D printed structures for a variety of tissue engineering applications ${ }^{19}$. Ideally, these structures emulate key physical and molecular features of the native extracellular matrix (ECM) composed of particularly complex fibrous structures in order to facilitate cell attachment, proliferation and differentiation, and ultimately result in new tissue growth. In line with this, chitosan a natural polycationic linear polysaccharide has been prevalently used in tissue engineering applications largely due to its structural similarity to glycosaminoglycans (GAGs), a major component of the ECM ${ }^{20,21}$. Chitosan-containing structures have been routinely fabricated through chemical or physical cross-linking of this polymer, as chitosan is a highly water-soluble polymer ${ }^{22}$. Given the cationic nature of chitosan, the most facile way to cross-link this polymer is by using ionic interactions, preferably by using a secondary polymer with anionic charges ${ }^{23}$. Along these lines, the biopolymer gellan gum is an anionic extracellular bacterial polysaccharide produced in high yield by the non-pathogenic strain Sphingomonas elodea ${ }^{24}$, and scaffolds composed of chitosan and gellan gum has been already reported in the literature ${ }^{25,26}$. Nevertheless, given the fibrous structure of the ECM, it would be ideal to make structures from these polymers, which has not been fulfilled yet. The structures are mainly fabricated using melt or solution electrospinning techniques, which are currently limited by the choice of implemented biopolymers - as polyelectrolyte complexes (PEC), for example, do not yield continuous fibers due to great charge repulsion and excessive plastic stretching of the electrospinning jet ${ }^{27}$. To address this challenge, a microfluidic system that would allow for the fabrication of aligned microstructures would be ideal for tissue engineering applications 28,29 .

The microfluidics field has developed rapidly over the past few decades, however the biological area has been relatively slow in adopting this technology with fabrication techniques often focusing on conventional approaches ${ }^{30}$. Recently, this field has increasingly switched from molded chipsets to 3D printed systems due to its capacity to form more complex geometries and at a reduced time from concept to device ${ }^{31}$, along with a 
range of functional elements that have already been reported ${ }^{32}$. In line with this, there has been a proportional rise in the uptake of microfluidic techniques by the biological field ${ }^{33}$. For biomaterials and their fabrication, Guzzi and Tibbitt very recently reviewed 3D printing of biomaterials and argued that 'new technologies are needed to produce medical materials with suitable mechanical and biochemical properties' ${ }^{34}$. A number of works outline the advantages of 3D printed microfluidics particularly in controlling materials' behaviour to form new biomaterials ${ }^{35-38}$. For instance, a recent review by Sun et al. ${ }^{39}$ details the merits of combining 3D printing and microfluidics that include: programmed flow control for stable microenvironment maintenance, mimicking functionality and extending cell viability, detectable generation of tissue/organ-level structures, etc.

In light of the above account of material selection for biomaterial formation and the fabrication capabilities via 3D printed microfluidics, the challenge herein is to develop a fabrication approach for guiding the self-assembly of two oppositely charged polyelectrolytes into hydrogel structures. For progression towards cell work, the biomaterial in question is to be mechanically strong, but not so that it will hinder cell attachment. As a result, the chosen fabrication approach involves the combination of two cross-linking mechanisms, ionically first during guided mixing followed by in-situ photo-crosslinking (an approach recently highlighted by Galarraga [34]) to further tune the mechanical properties to desirable strengths - in our case to replicate the structural hierarchy of collagen fibers. Many studies have demonstrated the benefits of cell attachment to microstructures, e.g. melt electro-written scaffolds ${ }^{40,41}$. However, challenges still exist in the fabrication of hydrogel PEC bundles, particularly in regards to controlled fabrication methods, e.g. guided flow and tunable/multiple crosslinking mechanisms ${ }^{42-45}$. A similar approach has been proposed by Sant et al. ${ }^{46}$, where they demonstrated the crosslinking and cell attachment capability for PEC fiber bundles using a standard microfluidic chip arrangement. We further demonstrate that this approach can be adapted for 3D printed microfluidic channels (as discussed previously) ${ }^{29}$ as well as incorporate an in-situ UV crosslinking mechanism [34] to better strengthen the PEC structure.

The scope of this study is therefore to develop a multi-stage fabrication technique to form aligned and robust microstructures and establish the technology capable of achieving this via $3 \mathrm{D}$ printed components. The proposed method is by (a) extruding these materials through 3D printed microfluidic channels and control the microstructure's formation via guided compaction, (b) cross-linking the compacted material in-situ to reinforce the microstructure, and (c) depositing the formed microstructures onto a substrate using a three-axis positioning platform to minimise affecting factors such as manual handling by placing the microstructures on a substrate in a spatially controlled manner. We have specifically chosen methacrylated gellan gum (GG-MA, a polyanion) ${ }^{47}$ and chitosan (CHT, a polycation) as our biomaterial ink, distinct from a bioink ${ }^{48}$, since the natural poly-electrostatic complexation of these two materials allows for the formation of polyelectrolyte complex (PEC) microstructures ${ }^{46}$. Moreover, GG-MA is a well established anionic polymer in biomedical engineering capable of being modified/tuned based on its methacrylation ratio and more importantly it is this polymer that permits the proposed two crosslinking mechanisms to take place. Lastly, physical characterisation of the formed fibers took place in order to investigate their properties and therefore the suitability of the proposed bio-fabrication technique. 


\section{Experimental}

\subsection{Materials and Characterisation}

\subsubsection{Materials Preparation}

High molecular weight CHT (Mw 310-400 kDa, 75-85\% degree of deacetylation) was purchased from Sigma-Aldrich (USA) and low-acyl gellan gum (GG) (Gelzan ${ }^{\mathrm{TM}}$ (CM), Mw 1,000 kDa) was available from CP Kelco (USA). All GG was purified to remove divalent cations using an established method ${ }^{49}$. The purified GG was modified with methacrylate groups in order to allow chemical crosslinking of the gel via UV irradiation. Briefly, $1 \%(\mathrm{w} / \mathrm{v})$ of GG was fully dissolved in deionized water at $90{ }^{\circ} \mathrm{C}$ for $30 \mathrm{~min}$, and then monitored until cooled to $50{ }^{\circ} \mathrm{C}$. Subsequently, $4 \%$ (v/v) of methacrylic anhydride (MA) was added and kept in a homogeneous state by means of an automated stirring system for $6 \mathrm{~h}$ at $\sim \mathrm{pH}$ 8.0. This was achieved by adding $5 \mathrm{M} \mathrm{NaOH}$ in a dropwise fashion to the solution resulting in a cloudy suspension. The modified GG-MA solution was segmented into $50 \mathrm{~mL}$ tubes and centrifuged at 4,400 rpm for $5 \mathrm{~min}$ at $21{ }^{\circ} \mathrm{C}$ to remove excess MA. The clear solution was poured directly into an excess of 3 times the volume of cold acetone to precipitate the modified GG-MA at $-20{ }^{\circ} \mathrm{C}$ overnight. The following solution was re-centrifuged at 7,500 rpm for 5 min at $21{ }^{\circ} \mathrm{C}$, the GG-MA precipitate was collected and excess acetone was removed. The GG-MA precipitate was resuspended in at least $50 \mathrm{~mL}$ of deionized water and heated to $37^{\circ} \mathrm{C}$ under constant stirring for 30 min to ensure complete dissolution. The following GG-MA solution was then purified by dialysis via a similar method for at least 7 days against distilled water maintained at $30{ }^{\circ} \mathrm{C}$ to ensure complete removal of any residual MA and acetone. Water was exchanged at least 3 times per day until a clear solution was evident. All the batches were lyophilized and stored in a dry place under dark conditions. The degree of methacrylation of GG-MA, defined as the number of methacrylate groups attached to GG divided by the number of hydroxyl groups of unreacted GG, was calculated by integrating the peak at $1.45 \mathrm{ppm}$ from the methyl group of the rhamnose unit and double bound protons of the methacrylate group (peaks at $\sim 6.20, \sim 5.77 \mathrm{ppm}$ ), which was calculated to be $13 \%$. Subsequently, CHT and as-prepared GG-MA were dissolved $(2 \% \mathrm{w} / \mathrm{v})$, respectively, in an aqueous solution of acetic acid ( $1 \%$ $\mathrm{v} / \mathrm{v}$ ) and in deionized water under constant stirring at $50{ }^{\circ} \mathrm{C}$ for $10 \mathrm{~min}$. A photo-initiator (Lithium phenyl-2,4,6-trimethylbenzoylphosphinate (LAP)), was added $(0.12 \% \mathrm{w} / \mathrm{v})$ to GG-MA for chemical crosslinking and kept under dark conditions prior to use. All other reagents were purchased from Sigma-Aldrich unless specifically mentioned otherwise.

\subsubsection{Rheological Characterisation}

GG-MA and CHT hydrogels (both alone and combined) were prepared and tested using an AR G-2 rheometer (TA instruments, USA). The combined hydrogel was extruded directly onto the stage to closely replicate structure alignment inside the hydrogel system. In addition, a cone-plate geometry (core diameter: 40 $\mathrm{mm}$; angle $1^{\circ}$; gap $30 \mu \mathrm{m}$ ) was selected due to the remaining fluid-like properties. Strain and frequency sweeps of $0.1-1000 \%$ and $0.1-1000 \mathrm{~Hz}$, respectively, were performed to assess the viscoelastic region. Following, the shear thinning effect and viscosity was evaluated via shear rates ranging from 0.1 to $1000 \mathrm{~s}^{-1}$ at $25{ }^{\circ} \mathrm{C}$. 
Temperature sweeps were performed on the GG-MA to establish the sol-gel transition state, storage modulus and loss modulus were measured as a function of temperature from 5 to $40{ }^{\circ} \mathrm{C}$ with a heating rate of $2{ }^{\circ} \mathrm{C} / \mathrm{min}$, frequency $1.0 \mathrm{~Hz}$ and fixed strain of $0.1 \%$. Oscillatory time sweeps were conducted across 20 min to quantify any change in the storage modulus and loss modulus. All tests were performed with a solvent trap to prevent water evaporation and repeated in triplicate with presented data representing the mean of each test and corresponding standard deviation.

\subsubsection{Crosslinking Kinetics by in-situ Rheology}

In order to establish the behaviour of the two materials (GG-MA and CHT) in combination (GG-MA:CHT) during fabrication, it was necessary to measure their kinetics under different photo-initiator concentrations for subsequent transfer to the control system. This was investigated by monitoring the real-time storage modulus upon UV exposure, using an Omnicure ${ }^{\circledR}$ LX400 LED UV Spot Curing System, (Lumen Dynamics, Canada) during an in-situ rheology time sweep using an Anton Paar MCR 301 Rheometer (Physica). A quartz stage was utilised, to allow UV light (from beneath) to penetrate through to the sample at a fixed distance of $5 \mathrm{~cm}$. The frequency was fixed at $1 \mathrm{~Hz}$ and strain set to $0.1 \%$. All the samples were exposed to UV light $\left(400 \mathrm{~nm}, 15.3 \mathrm{~mW} / \mathrm{cm}^{2}\right)$ for at least 60 seconds or until complete crosslinking had occurred while their modulus was measured in real-time; baseline measurements were recorded for 60 seconds prior to UV exposure, which was continued until a plateau was evident.

\subsection{Fabrication System}

\subsubsection{Microfluidic Nozzle}

The target function of the microfluidic nozzle was to allow for the two materials (CHT and GG-MA) to independently flow through their respective channel and gradually ionically cross-link at a mixing stage to enable polyelectrolyte complex formation to take place in a controlled linear manner. Immediately following this, the material was irradiated to enable cross-linking to take place resulting in robust structures emanating from the nozzle tip (figure 1). First, the materials were loaded into standard $5 \mathrm{~mL}$ disposable syringes. The inlets of the microfluidic system were designed with reciprocal/matching luer lock connections and placed at a distance to each other $(>27 \mathrm{~mm}$ ) to avoid interference during locking, i.e. rotation of the barrel flanges. Second, the materials flow along their respective channels $(\varnothing=800 \mu \mathrm{m})$ until they meet at the mixing stage $(\varnothing=800$ $\mu \mathrm{m})$ introduced to each other at the smallest angle possible during the fabrication process (i.e. an angle of $15^{\circ}$ ) and allowing self-assembly of structures to take place naturally. The microfluidic sub-system also featured placement of three (3) LEDs to ensure that cross-linking occurred through a 3 point surrounding strategy. The design was fabricated using a Stereolithography (SLA) printer (Form 2, Formlabs) using clear resin material (RS-F2-GPCL-04, Formlabs), which ultimately allowed for cross-linking to take place via the irradiance through the clear walls surrounding the mixing channel. 


\subsubsection{Extrusion Mechanism}

The next stage was to develop a mechanism that could: (a) hold all necessary components in static isolation, (b) mount onto the stage, and (c) extrude the two materials via their syringes into the microfluidic sub-system (nozzle). Figure 1 presents the design assembly of the extrusion system. The mechanism functions by means of the motor (Nema 11, non-captive, linear stepper motor) moving the lead screw, which enables direct translation of the carriage. In turn this pushes the syringe plungers into their barrels and forces the materials into the microfluidic nozzle. The extrusion design featured slots that fixed the barrell flanges in place while the carriage approaches/retracts. Finally, the brackets allowed for mounting on the 3-axis linear stage. The entire mechanism (holder and carriage) was fabricated on a commercial FDM printer (uPrint plus by Dimension, Stratasys) using ABS material (ivory colour).

\subsection{3. $\quad$ Stage Design and Assembly}

To (a) avoid the issue of manual handling, (b) have a means to controllably/reproducibly deposit the structures on a substrate in a controlled manner, and (c) have inherent access to stepper motor functionality, a 3D stage was developed. Figure 1 presents multiple views of the assembled printer. The stage (ME2, Me3D Pty Ltd) fulfilled the requirements for 3D movement in the denoted $\mathrm{X}, \mathrm{Y}$ and $\mathrm{Z}$ directions. The thermoplastic heating element was replaced by the developed extrusion control system. Additionally, the base was replaced with one that could accommodate three glass slides, which was fabricated from laser cutting (Laser Plotter, Universal Laser Systems) three designed pieces from $3 \mathrm{~mm}$ thick white perspex. The extrusion stepper motor was controlled by a PCB control board (Ultimaker 1.5.7) driven by firmware (Marlin). Considering the extrusion rate of the materials was an important factor for structure formation, a calibration routine took place to establish the relationship between the steps per $\mathrm{mm}$ and distance travelled by the carriage. This was achieved by means of measurements via Vernier Calipers and directed movement intervals by the control software (MatterControl). The ability to control the operating software through 'G-code' allowed for motorised axes to be translated according to a certain set of parameters, namely defining the location to which it should move as well as the speed and path it should take. Moreover, the feed rate $(\mathrm{mm} / \mathrm{s})$ of the mechanically driven piston was capable of being optimised to match the flow rate required for the adequate extrusion and formation of fiber bundles of interest. 


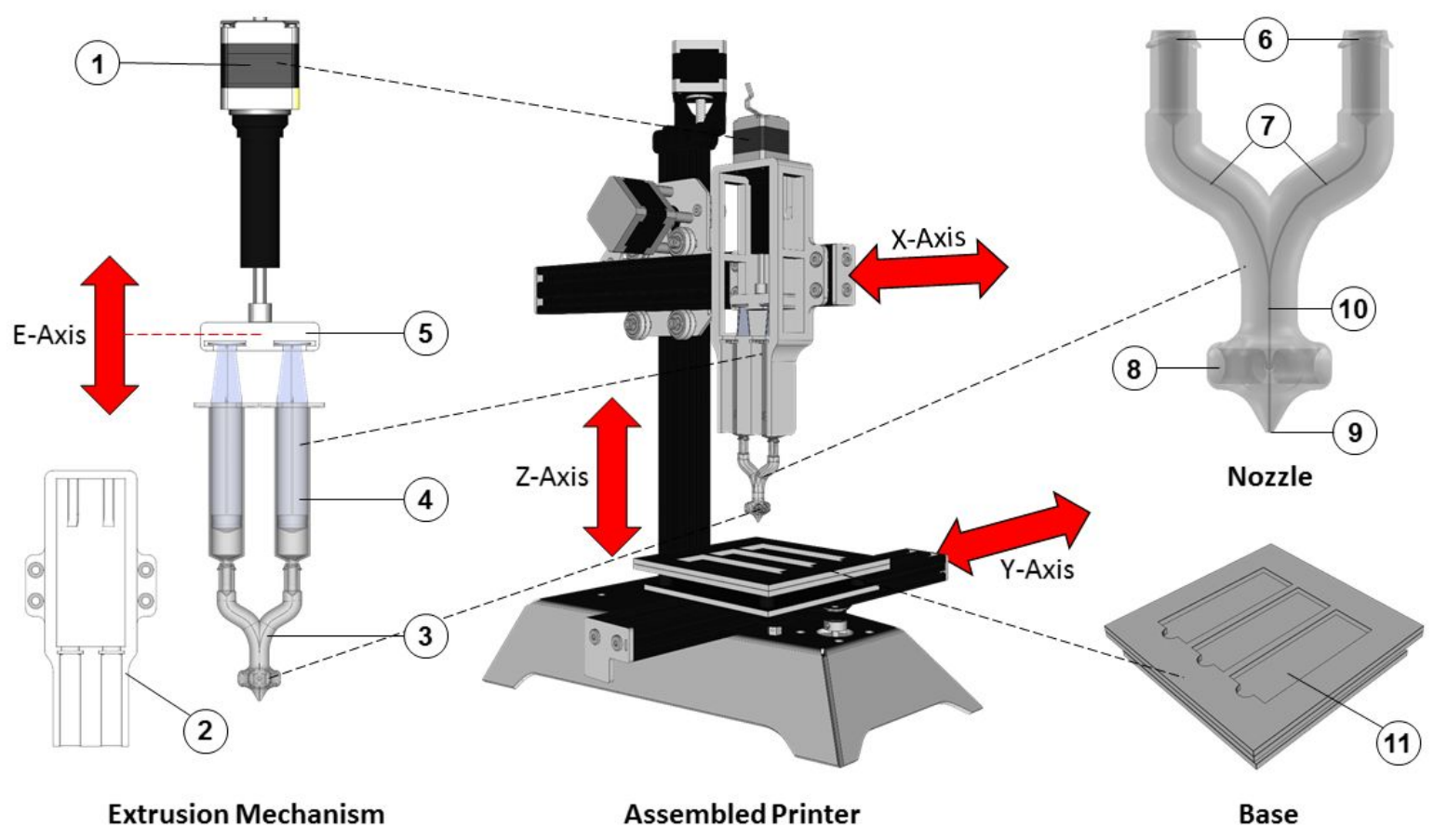

Figure 1. CAD design of the structure fabrication system. (1) Non-captive linear stepper motor. (2) Extrusion mechanism mounting system. (3) Nozzle. (4) Syringes. (5) Carriage. (6) Luer lock connections for securely attaching the syringes. (7) Fluidic channels. (8) Mounting points for the 3 LEDs. (9) Nozzle tip. (10) Mixing/compacting channel - $\varnothing=800 \mu \mathrm{m}$. (11) Base - glass slide holder.

\subsection{Printability}

\subsection{1. $\quad$ Hydrogel Structure Bundle Fabrication and Printing Sequence}

Fabrication of the aligned hydrogel structure bundles were performed with the custom-designed microfluidic nozzle and extrusion mechanism, as previously described. Both CHT and GG-MA inks were loaded into separate syringes and attached to the microfluidic nozzle device. Table 1 summarises the fabrication parameters of the system, see ESI Section S2 for extrusion system calibration. At a constant feed rate $(60 \mu \mathrm{m} / \mathrm{s})$ the inks were directed into each inlet of the microfluidic device where the two channels merge and initiated structure crosslinking. A UV intensity of $2.6 \mathrm{~mW} / \mathrm{cm}^{2}$ and wavelength of $395 \mathrm{~nm}$ was applied from each of the LEDs (UV5TZ-395-15 Bivar) to penetrate through the micro-channel walls measured with a LED light meter (Lumen Dynamics Omnicure LM2011/LS100 LED Light Meter System). The structure extrusion was maintained at a working distance of $0.2 \mathrm{~mm}$ between the nozzle and stage, with a single strand extrusion distance of 10-30 $\mathrm{mm}$.

Table 1. Hydrogel structure bundle fabrication parameters.

\begin{tabular}{|l|c|c|}
\hline \multicolumn{1}{|c|}{ Parameter } & Value & Unit \\
\hline Feed Rate & 60 & $\mu \mathrm{m} / \mathrm{s}$ \\
\hline Syringes Size & 3 & $\mathrm{cc}$ \\
\hline Microchannel Diameter & 800 & $\mu \mathrm{m}$ \\
\hline
\end{tabular}




\begin{tabular}{|l|c|c|}
\hline Output Current Pot Control & 600 & $\mathrm{mV}$ \\
\hline Microstep Resolution & Sixteenth & Steps \\
\hline Extrusion Height & 0.2 & $\mathrm{~mm}$ \\
\hline Microstructure Extruded Length & 30 & $\mathrm{~mm}$ \\
\hline
\end{tabular}

\subsubsection{Optical and SEM Microscopy}

Optical microscopy was employed using a LEICA M205A optical microscope to measure the average diameter for all GG-MA:CHT microstructure bundles and used to calculate the structure volume over the fixed structure length of 10-30 mm. This method assumed a consistent structure cross-section by calculating an average for each structure diameter over multiple randomly selected points $(\mathrm{n}=5)$. SEM microscopy was carried out using a JEOL JSM-7500FA Field Emission Electron Microscope (FE-SEM) to further investigate the structure diameter and morphology. Hydrogel structure bundles were flash-frozen in liquid nitrogen and freeze-dried for $72 \mathrm{hrs}$ before being sputter-coated with platinum (Pt) using an Edwards sputter-coater (coating thickness; $10 \mathrm{~nm})$. The images were captured at $15 \mathrm{kV}$ or $20 \mathrm{kV}$.

\subsubsection{Mechanical Properties of Printed Structures}

The mechanical properties of the as-prepared microstructure bundles were investigated using an EZ-L Mechanical Tester (Shimadzu, Japan). Freshly prepared structure bundles or overnight dried fibers ( $n=3)$ were used to determine the mechanical properties of fibers in wet and dry state, respectively. A gauge length of 10 $\mathrm{mm}$ was marked and used with load cells of 10 or $50 \mathrm{~N}$, for wet and dry fibers,respectively. The structures were stretched at a constant strain rate of $2 \mathrm{~mm} / \mathrm{min}$ until breaking point was achieved. For structures tested in a wet state, silicon was glued to the ends of wool string and placed in DI water for $10 \mathrm{~min}$ to induce swelling ${ }^{50}$. Optical images of each structure were captured to accurately measure their diameter to calculate the cross-sectional area. Due to an irregular surface, diameters were averaged over multiple points $(n=5)$. The stress (MPa) was calculated from the output data of applied force $(\mathrm{N})$ divided by the cross-sectional area and percentage elongation (strain) was obtained from the displacement using $L / L_{0} \times 100$, where $L_{0}$ was the original length $(10 \mathrm{~mm})$ and $L$ was the immediate length of the sample. The Young's modulus, ultimate stress and strain were determined from the stress-strain curve.

\section{Results and Discussion}

\subsection{Fabrication System}

The custom-design fabrication system can be seen in figure 2. All the elements of the CAD system previously explained were realised as discussed and subsequently assembled. The controlled movements of the $\mathrm{X}, \mathrm{Y}$ and $\mathrm{Z}$ axes in addition to the extrusion mechanism was possible by means of a motor control board (Ultimaker 1.5.7) and programmed with open-source firmware (Marlin, 1.0.1). Control of the LEDs for 
cross-linking was achieved by applying $3.4 \mathrm{~V}$ in parallel arrangement, supplying $\sim 18 \mathrm{~mA}$ to each LED. This was based on the intensity of the UV light used for rheological measurements using a light meter (Lumen Dynamics Omnicure LM2011/LS100 LED Light Meter System) measuring $15.3 \mathrm{~mW} / \mathrm{cm}^{2}$ held at a distance of 5 $\mathrm{cm}$ from the sample. Each LED resulted in $2.6 \mathrm{~mW} / \mathrm{cm}^{2}$ - cumulatively $7.8 \mathrm{~mW} / \mathrm{cm}^{2}$ ( $3 \mathrm{LEDs}$ ), i.e. half the relative intensity. However, by mounting the LEDs directly at the walls (300 $\mu \mathrm{m}$ thick) and reducing the amount of material and matching the flow rate, it was sufficient intensity to enable cross-linking.

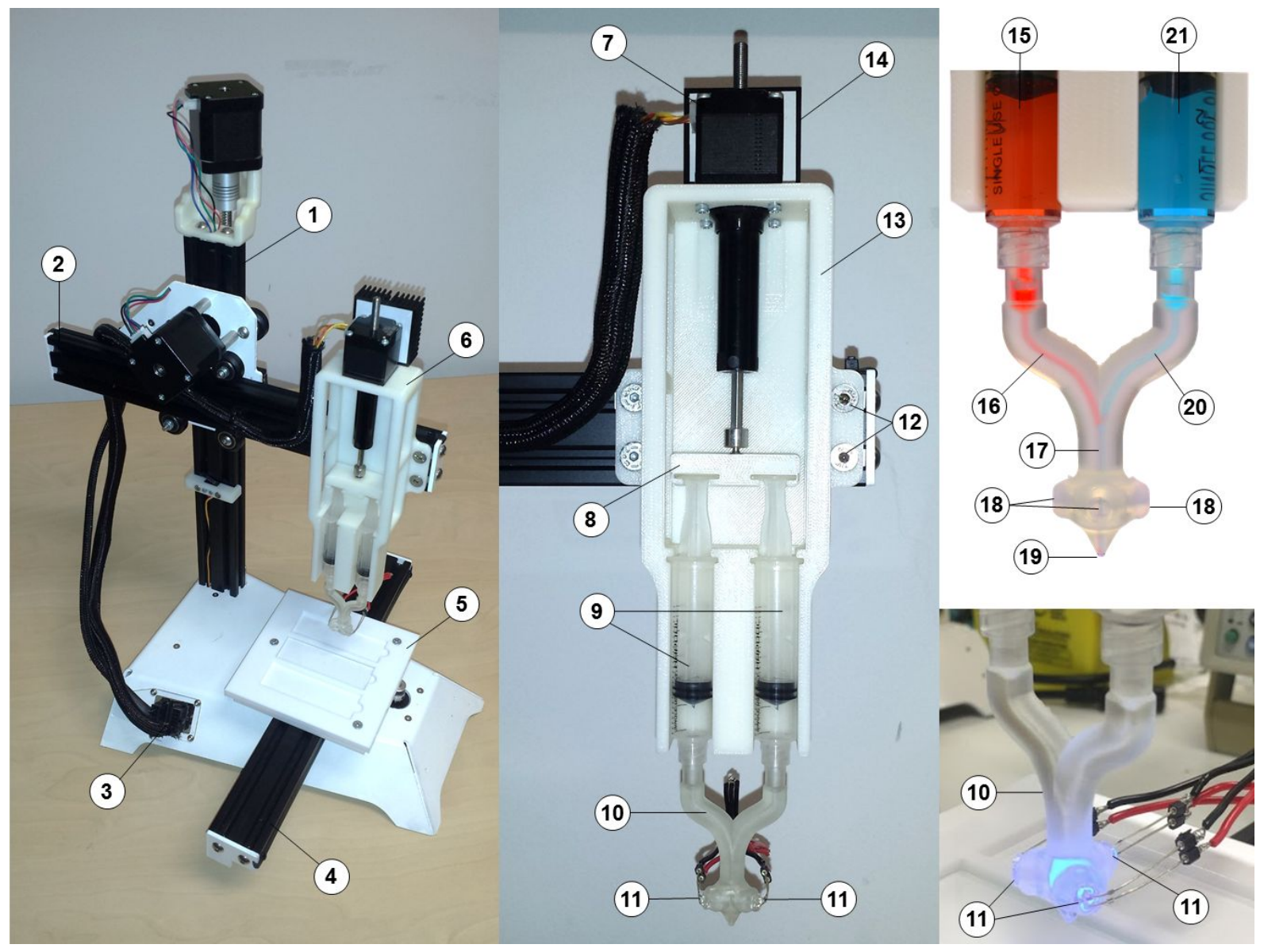

Figure 2. Captured and labelled photos of the assembled printer (left) with the extrusion mechanism (middle), the microfluidic nozzle (top right), and the nozzle with the LEDs illuminating (bottom right). (1) Z-Axis. (2) X-Axis. (3) Control wiring for the X-Axis and Extrusion Mechanism motors. (4) Y-Axis. (5) Printing base. (6) Extrusion Mechanism. (7) Nema 11 non-captive linear stepper motor. (8) Carriage. (9) Syringes. (10) Nozzle. (11) LEDs. (12) Mounting brackets. (13) Housing. (14) Heat sink. (15) Left syringe - red representing GG-AM. (16) Left fluidic channel. (17) Mixing channel. (18) LED placements. (19) Nozzle tip. (20) Right fluidic channel. (21) Right syringe - blue representing CHT.

\subsection{Material Characterisation}

In order to explore a number of primary factors affecting structure formation, the materials underwent a number of analyses using state-of-the-art instrumentation to ensure material characterisation. The factors include flow behaviour and crosslinking kinetic. The rheological profile of the polymers before and after cross-linking were established to provide insight into the complexation of these structures through microfluidic channels. The polymer concentration of $2 \% \mathrm{w} / \mathrm{v}$, ratio 1:1 (GGMA:CHT) and the nozzle was selected for showing the printed structure formation during the microfluidic study. Therefore for all rheological tests $2 \% \mathrm{w} / \mathrm{v}$ GG-MA:CHT (1:1) 
was investigated. Figure 3 presents rheological analysis of the materials with discussions in the following subsections.
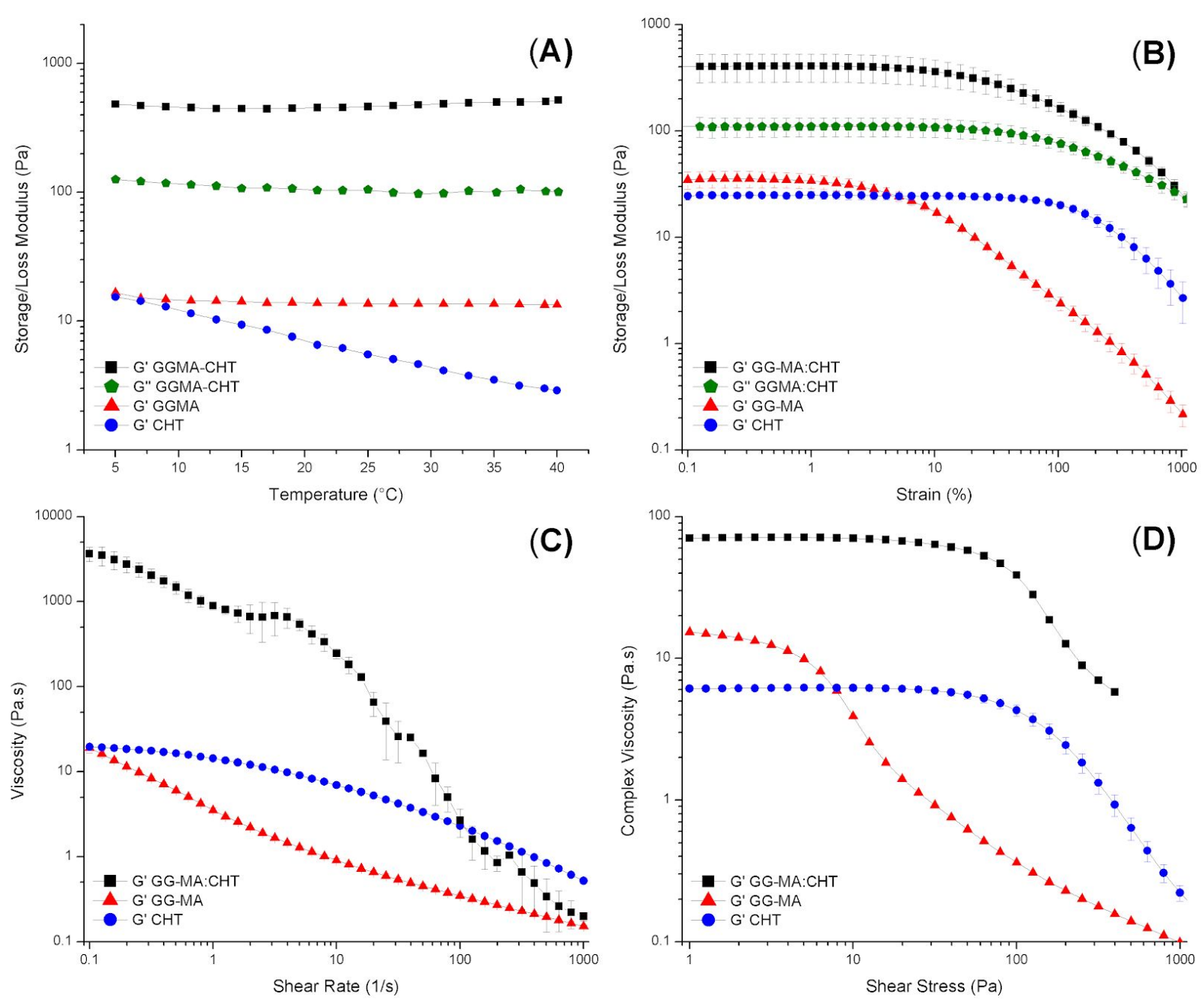

Figure 3. Rheological characterisation of $2 \% \mathrm{w} / \mathrm{v}$ GG-MA (in deionized water), $2 \% \mathrm{w} / \mathrm{v}$ CHT (in an aqueous solution of acetic acid, 1\% v/v), and GG-MA:CHT the complexed hydrogel solution. Data points represent the average values and the error bars are the standard deviation $(\mathrm{n}=3)$. (A) Temperature sweeps from 5 to $40^{\circ} \mathrm{C}$ with a constant heating rate of $2{ }^{\circ} \mathrm{C} / \mathrm{min}$ ramp, a frequency of $1 \mathrm{~Hz}$ and strain of $0.1 \%$. (B) Strain sweep for all corresponding hydrogel precursors at room temperature $\left(25^{\circ} \mathrm{C}\right)$. (C) Flow profile showing the materials' shearing-thinning effect during shear rate sweep at constant room temperature $\left(25^{\circ} \mathrm{C}\right)$. (D) Yield stress measurements. Data points represent the average with error bars the standard deviation $(n=3)$.

\subsubsection{Effect of Temperature on the ViscoElastic Properties of the Printed Structures}

Figure 3a presents the storage and loss moduli of GG-MA, CHT and as-extruded structures from 5-40 ${ }^{\circ} \mathrm{C}$. At $2 \% \mathrm{w} / \mathrm{v}$ GG-MA it was demonstrated that effectively no dependence on temperature, as the storage modulus $\left(\mathrm{G}^{\prime}\right)$ remained consistent at $14 \pm 0.77 \mathrm{~Pa}$, while CHT showed swift deformation and storage modulus almost half at $40{ }^{\circ} \mathrm{C}$. This is in agreement with previous studies, where the dynamic viscosity decreased over time due to the rapid dissociation of CHT molecular network in the solution when its activation energy threshold is achieved ${ }^{51}$. As seen in figure 3a, the storage modulus was stable over the temperature range from 5 to $40{ }^{\circ} \mathrm{C}$. This is consistent with previous reports that the formed PECs had better stability (against temperature and shear stress) 
than each individual component (GG-MA and CHT in this case) $)^{52}$. Conversely, these results support the criteria that no direct temperature control is required for the developed printer setup. Consequently, the subsequent printing experiments were conducted at room temperature.

\subsubsection{Mechanical Shear \& Strain}

Considering that the materials will be undergoing mechanical shear and strain effects, analysis of the materials behaviour was necessary. The viscoelastic region for GG-MA was found to reach $\sim 1 \%$ strain, before undergoing deformation, indicating that it is still relatively liquid-like at $2 \% \mathrm{w} / \mathrm{v}$ polymer concentration. While $\mathrm{CHT}$ at $2 \%$ w/v polymer concentration exhibited a much higher strain of $\sim 100 \%$ before its polymer network initiated deformation (figure $3 \mathbf{b}$ ). This is partly due to the selected high molecular weight of CHT and the high surface tension characteristic of $\mathrm{CHT}^{51}$. Figure $3 \mathbf{b}$ also shows the fibrous hydrogel system of GG-MA:CHT $(\sim 10 \%$ strain) following destruction of the polyelectrolyte complex network. Comparisons of these results demonstrate that once the hydrogel structure bundle is formed the strain is equilibrated to $10 \%$ strain and notably the storage modulus increases up to $400 \pm 2.74 \mathrm{~Pa}$, due to physical crosslinking between the polyanion and polycation.

A shear rate sweep was performed on each of the inks, in particular, the combined hydrogel structure bundle to confirm that the PEC structure holds a shear-thinning behaviour. Firstly, it was shown that both GG-MA and CHT demonstrated shear-thinning, beginning from $\sim 20$ Pa.s and decreasing as shear rate increases (figure 3c). The shear rate sweep for the hydrogel structure bundles were difficult to measure due to aligned structures becoming increasingly entangled upon high shear rates with a cone-plate geometry. Figure $3 \mathbf{c}$ also shows that the combined hydrogel was overall shear-thinning, however this is not a smooth behaviour due to the loss of alignment of respective structures on the rheometer. The final entangling of the structure network and a hydrogel sheet with effectively a non-aligned structure system was observed. Multiple tests were performed $(n=3)$ to observe this behaviour and the reproducibility thereof.

Following this, a yield stress measurement was conducted for all corresponding hydrogels to evaluate the minimal shear stress required to initiate the flow. Figure 3d shows that GG-MA requires the lowest applied stress ( $\sim 10 \mathrm{~Pa}$ ); this is expected due to it still being mostly liquid-like with a capacity to readily flow. However, CHT is much higher at $\sim 100 \mathrm{~Pa}$ applied stress to induce any sort flow of the polymer solution. This is anticipated by the fact CHT has a high surface tension value, however it has been shown that higher polymer concentrations result in a gradual lowering of the surface tension ${ }^{53}$. Figure $3 \mathbf{d}$ also shows that the applied stress required for the combined hydrogel structure bundle is similar to that of CHT. This suggests that GG-MA is being complexed with the CHT polymer chains and that excess CHT solution remains around the shell of PEC structure formation. It should be noted that materials obtained via chemical crosslinking, i.e. ionically only within the microchannel, were unable to retain the original shape after lifting from the glass slide after deposition. While Figure 3 shows an increase in mechanical strength with GG-MA:CHT when compared to GG-MA and CHT alone, it evidently requires a second stage (photo crosslinking) to tune the mechanical properties to an extent that the printed form can be retained. 


\subsubsection{Cross-linking Kinetics}

In identifying other parameters for the translation of this microfluidic approach to a 3D- printer set up, it has been reported that PEC hydrogels have poor hydrolytic stability after complexation ${ }^{26}$. This is reportedly due to their crosslinking being highly dependent on physicochemical interactions between each polymer. The next investigation was based on if the methacrylate groups from the GG-MA could be photocrosslinked prior to extrusion to maximise the stability and handling. This would enable the ability to create a highly stable network and allow for further strengthened hydrogel structures. To better understand the crosslinking kinetics within the microfluidic system and after the post extrusion process, the crosslinking kinetics of the combined polyelectrolyte structure bundle was assessed. Figure 4 shows an increase of storage modulus upon UV exposure (measured at $15.3 \mathrm{~mW} / \mathrm{cm}^{2}$ by Lumen Dynamics Omnicure LM2011) for 60 seconds. It can be seen that $0.06 \%(\mathrm{w} / \mathrm{v})$ LAP only reached $\sim 1,414 \mathrm{~Pa}$ after 740 seconds exposure to UV light. These results depict that, compared to GG-MA in the absence of UV irradiation, incorporation of the photoinitiator (followed by UV irradiation) allows for a higher level of chemical crosslinking within the hydrogel structure bundle due to formation of covalent bonds between the methacrylate groups on the back-bone of GG-MA. Most importantly, after UV irradiation, GG-MA:CHT complex showed a lower storage modulus when compared to GG-MA, which was due to dilution effect of GG-MA in the complex solution, as well as presence of CHT chains in the complex which interfered with covalent bonds formations ${ }^{49}$.

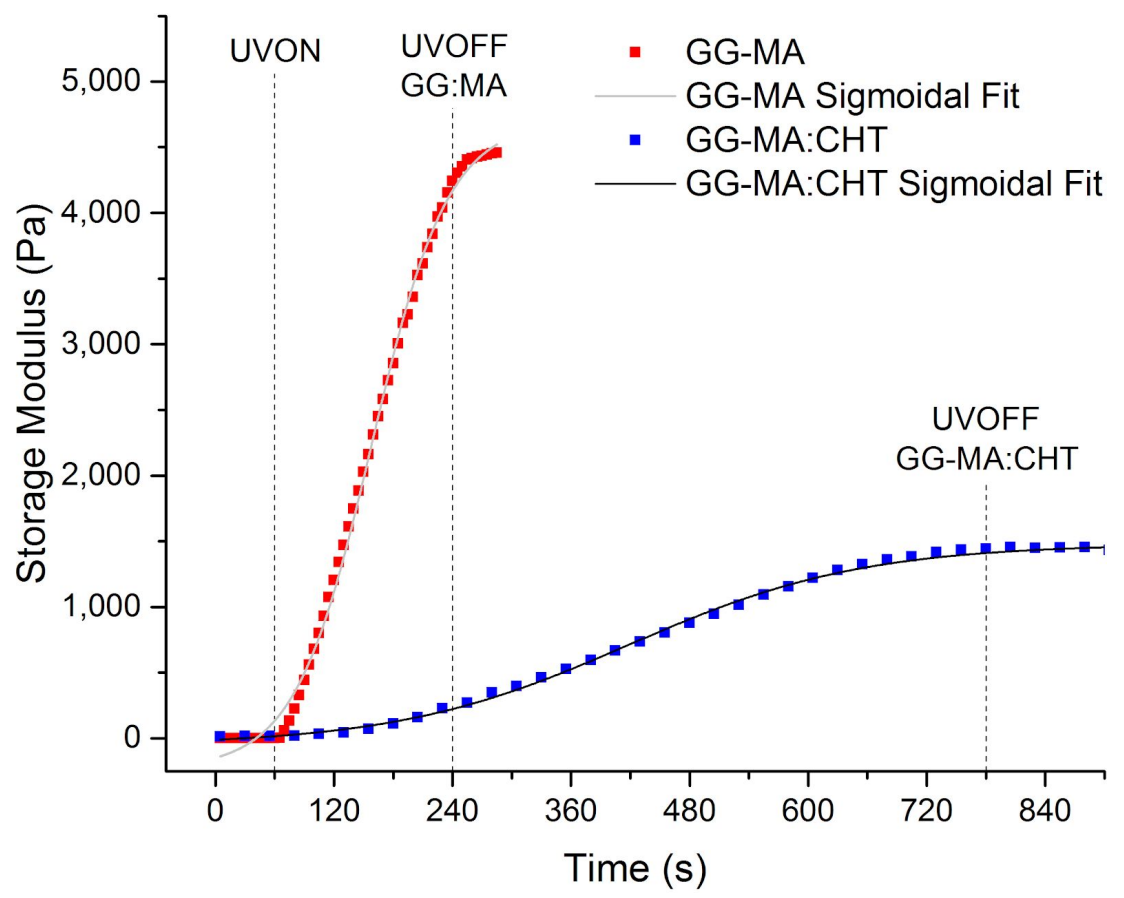

Figure 4. In-situ crosslinking kinetics of $2 \% \mathrm{w} / \mathrm{v}$ GG-MA and $2 \% \mathrm{w} / \mathrm{v}$ GG-MA:CHT hydrogel structure bundle. Each test was performed with $0.06 \% \mathrm{w} / \mathrm{v}$ LAP with the UV light source held at a distance of $5 \mathrm{~cm}$. The UV irradiance $\left(15.3 \mathrm{~mW} / \mathrm{cm}^{2}\right)$ was initiated at 60 seconds for both GG-MA and GG-MA:CHT and exposed for a duration of: 2 mins for GG-MA (off at $240 \mathrm{~s}$ ) and $12 \mathrm{mins}$ for GG-MA:CHT (off at $780 \mathrm{~s}$ ). The lines represent a sigmoid fit to the data for GG-MA (grey, $\left.\mathrm{R}^{2}=0.99784\right)$ and GG-MA:CHT (black, $\left.\mathrm{R}^{2}=0.99795\right)$. 


\subsection{Hydrogel Printed Micro Structure Fabrication and Analysis}

\subsubsection{Printed Structure Formation}

Based on the rheometry experiments conducted earlier (Section 3.1), fibrous hydrogel microstructures were successfully fabricated by extruding 2\% w/v solutions of CHT and GG-MA, 1:1 ratio, through the designed printer nozzle using the microfluidic approach as discussed. The polyelectrolyte complexation was initiated when the polymers came in contact with each other, i.e. when one polymer contacted the other counter-charged polymer, and UV crosslinked prior to extrusion. The effects of increasing feed rate on the formed microstructure were observed and are presented in the ESI (Section S3). After extrusion these stabilised structures were deposited onto a glass slide via the 3D stage, while minimising manual handling effects and avoiding mechanical testing.
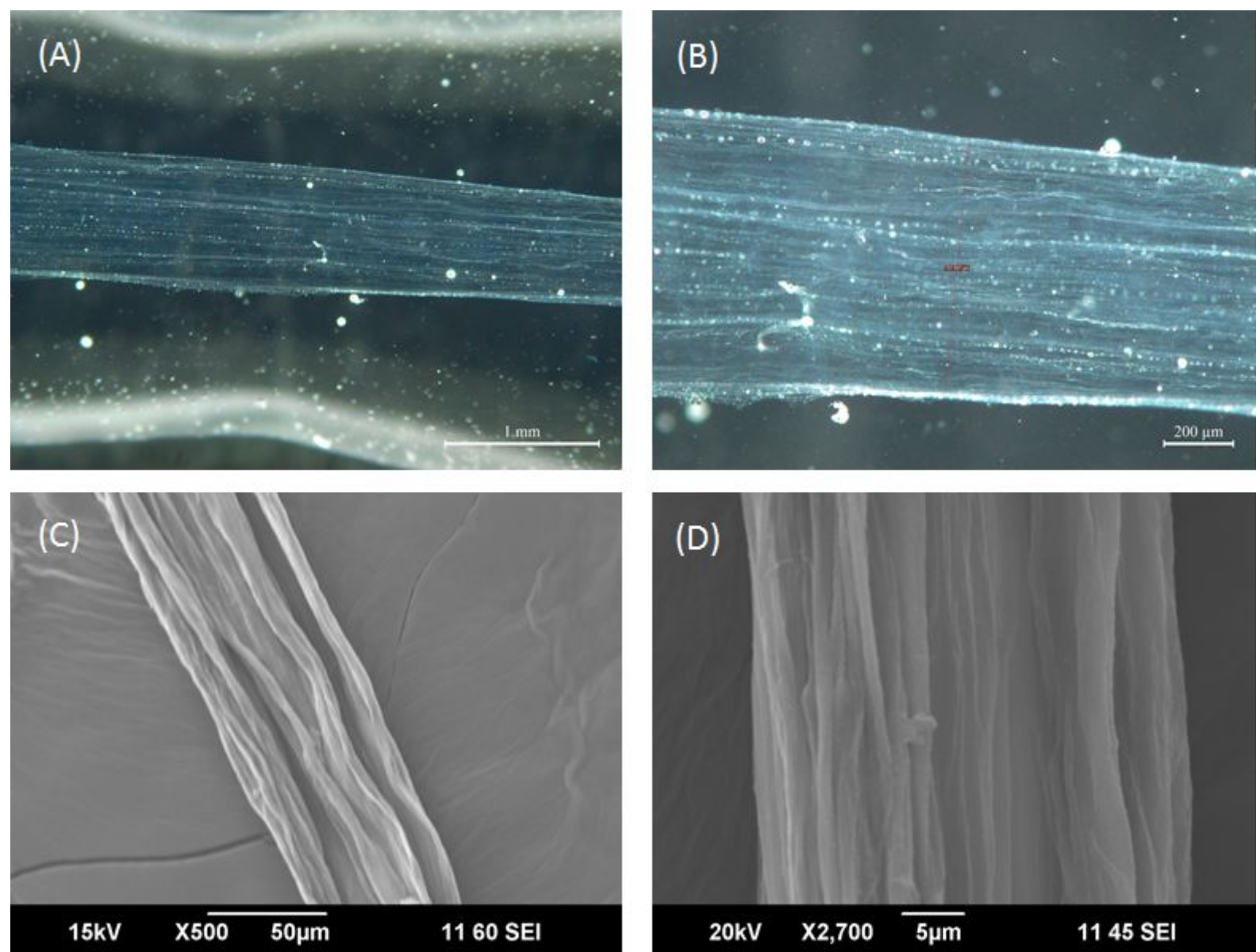

Figure 5. Light microscope and scanning electron microscope (SEM) images showing a compact aligned structure formation extruded. (A \& B) Light microscope images showing alignment of wet structures with 1:1 GG-MA/CHT. The corresponding structure diameters is $737.007 \mu \mathrm{m}$. (C \& D) Scanning electron microscopy of hydrogel fiber bundle (in dry state) showing multiple microstructures aligned parallel to each other. The figures show fiber bundle strands with an average fiber bundle diameter for the integration of both polymers and each microstructure $(\sim 1-2 \mu \mathrm{m})$ within the bundle. Each hydrogel was extruded from a microfluidic tip with an internal inner diameter of $1 \mathrm{~mm}$ and feed rate $3.63 \mathrm{~mm} / \mathrm{min}$.

Expelled water and excess gel was found surrounding the extruded structure bundles at $\sim 100 \mu \mathrm{m}$ on either side, this occurs when the oppositely charged structures bind along each polymer's backbone and push water out from 
between. With a structure diameter of $737 \mu \mathrm{m}$, this corresponds to the inner diameter of $800 \mu \mathrm{m}$ inside the nozzle. Once it was established that polyelectrolyte structure bundles could be achieved via printing single strands, the feed rate was evaluated to determine whether the structure diameter could be controlled based on the applied pressure and mixing of these two materials. Due to the complex and dynamic nature of this microfluidic system, partnered with two separate hydrogels, it is assumed that structure formation is related to the applied pressure between the two solutions. Therefore, conceptually, with increased feed rate, more gelation would occur allowing for future investigation into various tissue types, see ESI (Section S3) for further information.

As shown in figure 5 , a feed rate of $3.63 \mathrm{~mm} / \mathrm{min}(60.5 \mu \mathrm{m} / \mathrm{s})$ produced a fiber bundle with a diameter of $802 \pm 60 \mu \mathrm{m}(\mathrm{n}=22)$. It should be noted that when a reduced feed rate is used, large amounts of excess material does not undergo cross-linking within the structure bundle, see Section S3 in the ESI. This required its removal from the glass slide post printing. Interestingly, at both resolutions, striations can be observed under an optical microscope. Hypothetically, this corresponds to the longitudinal repetition of oppositely charged CHT and GG-MA polymer chains. Most importantly, these bundles showed an aligned fibrous structure. In natural collagen, these features occur as a result of the staggered repetition of tropocollagen molecules, linearly self-assembled by electrostatic interactions, and laterally packed through hydrogen bonds and hydrophobic forces $^{54,55}$.

Using scanning electron microscopy (SEM), the microscale structure characterisation confirmed that the structures involved a highly organised fibrilar bundle, similar to that observed in natural collagen structures. SEM shows that the fibrous hydrogel is made up of a series of micro-fibrils aligned in parallel and packed together in a tight bundle (figure $5 \mathbf{c \& d}$ ) after being extruded the designed printer nozzle. Figure 5 at the highest magnification revealed the morphology of fibrous hydrogel, indicating each micro-fibril size varied from 1-2 $\mu \mathrm{m}$. This is consistent with other studies ${ }^{26,46,56}$, further establishing that polyelectrolyte structure formation is a viable method for replicating the structural hierarchy of native collagen structures.

\subsubsection{Mechanical Properties}

Since collagen is responsible for providing high tensile strength to tissues, such as the cardiac skeleton of the heart ${ }^{57}$, the meninges of the brain ${ }^{58}$, the reticular layer of skin ${ }^{59,60}$, and the endomysium of muscle ${ }^{61,62}$, mechanical characterisation of the as-prepared hydrogel structure bundles was investigated. It was shown that the GG-MA:CHT structure bundles were mechanically strong both at its dry-stage and wet-stage as evident by obtained data for elastic modulus $(1,150 \pm 0.05 \mathrm{MPa}$ and $1.04 \pm 0.14 \mathrm{MPa}$, respectively) and tensile strength $(22,170 \pm 0.02 \mathrm{MPa}$ and $0.79 \pm 0.18 \mathrm{MPa}$, respectively) (figure 6 and table 2). The obtained results for fibers in wet-state were found to be similar to previous studies of forming PEC structures on PDMS microfluidic systems which obtained elastic modulus of approximately $2.81 \mathrm{MPa}$ and tensile strength of $1 \mathrm{MPa}^{46,63}$. The mechanical properties of PEC fibers ultimately depends on multiple factors including but not limited to molecular weight and corresponding concentration of each element (GG-MA and CHT), and degree of methacrylation of GG. Nonetheless, the significant difference between obtained mechanical properties in dry and wet states is due to a 
swelling phenomenon in hydrogels which yields polymeric networks with high water content that translates into weaker interchain molecular interactions ${ }^{23}$. Overall, obtained PEC fibers possessed good mechanical integrity, compared to similar studies, that might facilitate the application of such structures in tissue engineering applications.

Table 2. Mechanical properties of GG-MA:CHT in its dry and wet states (average \pm stdev).

\begin{tabular}{|l|c|c|}
\hline & Dry State & Wet State \\
\hline Modulus (MPa) & $1,150 \pm 0.05$ & $1.04 \pm 0.14$ \\
\hline Tensile Strength (MPa) & $22,170 \pm 0.02$ & $0.79 \pm 0.18$ \\
\hline Elongation (\%) & $3.12 \pm 0.96$ & $84.19 \pm 18.32$ \\
\hline
\end{tabular}
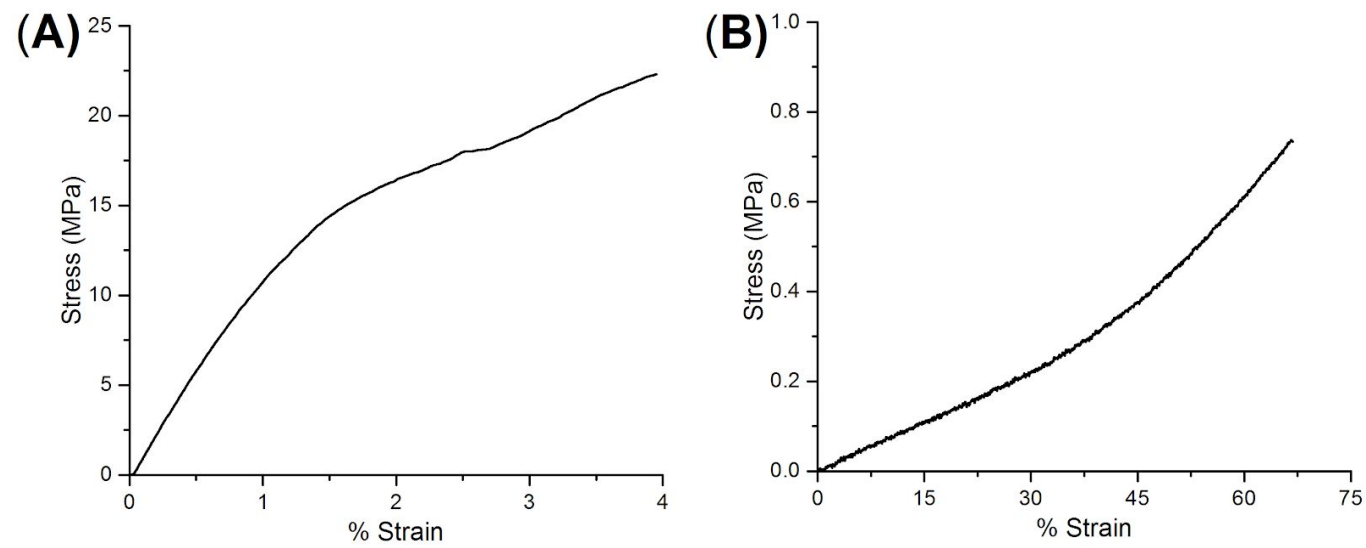

Figure 6. Mechanical characterisation of GG-MA:CHT hydrogel structure bundle. Tensile testing of stress-strain curves for structures in: (A) dry state and (B) wet state.

\subsection{Notes and Future Work}

Having successfully established the formation of the biomaterial and engineering factors, our subsequent study will explore the formation of 3D scaffolds and cell work. The findings of this study have demonstrated the fabrication of microstructures via 3D printed components, and therefore scope for printing higher dimensional structures, e.g. 3D scaffolds. For instance, the 3D stage has been developed and in place to provide controlled 3D movements. Additionally, the 3D printed microfluidic channels successfully formed functional biomaterials of aligned-structure hydrogel bundles via the designed extrusion system. Moreover, simultaneous control of the 3 translational axes and extrusion system were successfully shown via precise deposition onto a glass slide. Also, the feed rate has shown to influence the compaction taking place, which can be complimentary controlled by varying the intensity of the LEDs. Thus, the majority of elements are in place to progress to $3 \mathrm{D}$ scaffold 
construction and explore cell work. Overall, this work has revealed challenges to overcome, which constitute an interesting body of work in itself.

It has become clear in this study that the excess expelled during compaction is a necessary byproduct for successful extrusion, i.e. in order to supply a barrier between the compacted material and the channel wall. However, this brings additional challenges when moving forward towards 3D scaffold construction. In line with this, a substrate capable of anchoring to the depositing hydrogel will need addressing, possibly via an absorbing material or a grate with a vacuum to remove the excess. Subsequent layers will face an added challenge of fully adhering to the underlying layer(s) - we envisage an additional crosslinking mechanism as the LEDs have demonstrated photo-crosslinking capability and the rheometry has shown scope for a higher achievable storage modulus. Another aspect to address is continuous filament formation as stopping and starting the feed rate may lead to nozzle blockages. A cleaning station would be required for complex shapes. One option for investigating the effectiveness of structures is to mark the polymers with a dye and visualise the structure of the printed scaffold with laser or confocal microscopy. As cell staining will take place regardless for cell viability studies, this would be a more effective method to determine the compacting of the two polymers. Coupling this with image processing methods, a good indirect approximation of the complexation can be determined in addition to the extent of cell viability.

Since single hydrogel solutions possess challenges ${ }^{64}$, insofar that they become too soft or stiff, this approach provides mechanical strength in a soft hydrogel environment as well as directional cell attachment to structures for cells. We have implemented two cross-linking mechanisms to provide sufficient control of the biomaterials's strength into a spatially controlled system. While this study focused on the development of the engineering aspects using developed biomaterial ink as described by literature ${ }^{48}$. Further integration of cells into this system will expand Sant et al. and co-worker's findings who have previously demonstrated cell attachment with this material using human mesenchymal stem cells (hMSCs) encapsulated in a simple microfluidic chamber ${ }^{46}$. Moreover, establishing GG-MA gels alone do not provide cellular attachment and Arginylglycylaspartic acid (RGD) bio-functionalization is required to provide integrin binding sites for cell attachment along the fiber bundle. This will involve studies into cell viability to address the effects of mechanical pressure, compaction, and irradiation on the bioink during the fabrication process demonstrated in this study.

\section{Conclusions}

This study successfully developed a multi-stage fabrication technique to form aligned and robust microstructures and established the technology capable of achieving this via 3D printed components. The fabrication of aligned-microstructure hydrogel bundles through 3D printed microfluidics was successfully demonstrated in this study. We have described the potential of printing with PEC structures by utilising the electrostatic interactions between GG-MA and CHT. The natural linear self-assembly of collagen structure bundles could be replicated and are further stabilised by in-situ UV crosslinking. We have proposed a 
biofabrication approach utilising 3D printed microfluidic technology that can be well-controlled, enabling the fabrication of complex scaffolds. Moreover, the individual biomimetic hydrogel structure bundles exhibit significant mechanical properties, showing $1.04 \pm 0.14 \mathrm{MPa}$ elastic modulus which is comparable to the magnitude for collagen tissue found within the body. The SEM characterisation showed that these hydrogel structure bundles $(\sim 50 \mu \mathrm{m})$ are organised into a bundle of microfibrils. Our results show consistency with the literature and further build upon providing a solid biofabrication platform upon which further studies with different polymers and applications of these biomimetic structures can be explored. Potentially, one can use this technology to encapsulate neural cells into the hydrogel bundles, with the aim of guiding the axonal growth within the aligned micro-structure of these fibers ${ }^{65}$. Given the aligned nature of yielded fibers on this platform, it can also be used to develop muscle-inspired highly anisotropic structures for potential biomedical applications

66. Overall, this study is the first to explore GGMA:CHT microstructure fabrication by means of controlled in-line compaction and photo-crosslinking through 3D printed microfluidic channels.

\section{Acknowledgements}

This work has been supported by the financial support provided by the Australian Research Council (ARC) Centre of Excellence Scheme (CE140100012) and the Australian Government through the International Education and Training program in relation to project BIOFABrication for Future Manufacturing. Special thanks to CP Kelco U.S. Inc. for supplying the low-acyl gellan gum (GG) used in this study. The authors gratefully acknowledge the use of facilities at the University of Wollongong Electron Microscopy Centre and support of the Australian National Fabrication Facility (ANFF), Materials Node - specifically from Mr. Adam Taylor and Mr. Grant Barnsley. Also, the authors wish to acknowledge great input and insight from \&btS Dr. Holly Warren-Hunt on characterisation of the materials via rheology. 


\section{References}

(1) Murphy, S. V.; Atala, A. 3D Bioprinting of Tissues and Organs. Nature Biotechnology. 2014, pp 773-785. https://doi.org/10.1038/nbt.2958.

(2) Chia, H. N.; Wu, B. M. Recent Advances in 3D Printing of Biomaterials. Journal of Biological Engineering. 2015. https://doi.org/10.1186/s13036-015-0001-4.

(3) Chiulan, I.; Frone, A.; Brandabur, C.; Panaitescu, D. Recent Advances in 3D Printing of Aliphatic Polyesters. Bioengineering. 2017, p 2. https://doi.org/10.3390/bioengineering5010002.

(4) Hospodiuk, M.; Dey, M.; Sosnoski, D.; Ozbolat, I. T. The Bioink: A Comprehensive Review on Bioprintable Materials. Biotechnol. Adv. 2017, 35 (2), 217-239.

(5) Kačarević, Ž. P.; Rider, P. M.; Alkildani, S.; Retnasingh, S.; Smeets, R.; Jung, O.; Ivanišević, Z.; Barbeck, M. An Introduction to 3D Bioprinting: Possibilities, Challenges and Future Aspects. Materials 2018, 11 (11). https://doi.org/10.3390/ma11112199.

(6) Varkey, M. Current Challenges and Future Perspectives of Bioprinting. 3D Bioprinting in Regenerative Engineering. pp 359-373. https://doi.org/10.1201/b21916-16.

(7) Mandrycky, C.; Wang, Z.; Kim, K.; Kim, D.-H. 3D Bioprinting for Engineering Complex Tissues. Biotechnology Advances. 2016, pp 422-434. https://doi.org/10.1016/j.biotechadv.2015.12.011.

(8) Ligon, S. C.; Liska, R.; Stampfl, J.; Gurr, M.; Mülhaupt, R. Polymers for 3D Printing and Customized Additive Manufacturing. Chem. Rev. 2017, 117 (15), 10212-10290.

(9) Placone, J. K.; Engler, A. J. Recent Advances in Extrusion-Based 3D Printing for Biomedical Applications. Adv. Healthc. Mater. 2018, 7 (8), e1701161.

(10) Mistry, P.; Aied, A.; Alexander, M.; Shakesheff, K.; Bennett, A.; Yang, J. Bioprinting Using Mechanically Robust Core-Shell Cell-Laden Hydrogel Strands. Macromol. Biosci. 2017, 17 (6). https://doi.org/10.1002/mabi.201600472.

(11) Noor, N.; Shapira, A.; Edri, R.; Gal, I.; Wertheim, L.; Dvir, T. 3D Printing of Personalized Thick and Perfusable Cardiac Patches and Hearts. Advanced Science. 2019, p 1900344. https://doi.org/10.1002/advs.201900344.

(12) Mannoor, M. S.; Jiang, Z.; James, T.; Kong, Y. L.; Malatesta, K. A.; Soboyejo, W. O.; Verma, N.; Gracias, D. H.; McAlpine, M. C. 3D Printed Bionic Ears. Nano Lett. 2013, 13 (6), 2634-2639.

(13) Nagle, A. R.; Fay, C. D.; Xie, Z.; Wallace, G. G.; Wang, X.; Higgins, M. J. A Direct 3D Suspension near-Field Electrospinning Technique for the Fabrication of Polymer Nanoarrays. Nanotechnology 2019, 30 (19), 195301.

(14) Robinson T. M., Hutmacher D. W., Dalton P. D. The Next Frontier in Melt Electrospinning: Taming the Jet. $A d v$. Funct. Mater. 2019. https://doi.org/Accepted.

(15) Castilho, M.; van Mil, A.; Maher, M.; Metz, C. H. G.; Hochleitner, G.; Groll, J.; Doevendans, P. A.; Ito, K.; Sluijter, J. P. G.; Malda, J. Melt Electrowriting Allows Tailored Microstructural and Mechanical Design of Scaffolds to Advance Functional Human Myocardial Tissue Formation. Advanced Functional Materials. 2018, p 1803151. https://doi.org/10.1002/adfm.201803151.

(16) Nagle, A. R.; Fay, C. D.; Wallace, G. G.; Xie, Z.; Wang, X.; Higgins, M. J. Patterning and Process Parameter Effects in 3D Suspension near-Field Electrospinning of Nanoarrays. Nanotechnology 2019, 30 (49), 495301.

(17) Pedde, R. D.; Mirani, B.; Navaei, A.; Styan, T.; Wong, S.; Mehrali, M.; Thakur, A.; Mohtaram, N. K.; Bayati, A.; Dolatshahi-Pirouz, A.; et al. Emerging Biofabrication Strategies for Engineering Complex Tissue Constructs. $A d v$. Mater. 2017, 29 (19). https://doi.org/10.1002/adma.201606061.

(18) Dinoro, J.; Maher, M.; Talebian, S.; Jarfarkhani, M.; Mehrali, M.; Orive, G.; Foroughi, J.; Lord, M. S.; Dolatshahi-Pirouz, A. Sulfated Polysaccharide-Based Scaffolds for Orthopaedic Tissue Engineering. Biomaterials. 2019. https://doi.org/10.1016/j.biomaterials.2019.05.025.

(19) Gopinathan, J.; Noh, I. Recent Trends in Bioinks for 3D Printing. Biomater Res 2018, 22, 11.

(20) Talebian, S.; Mehrali, M.; Mohan, S.; Raghavendran, H. R. B.; Mehrali, M.; Khanlou, H. M.; Kamarul, T.; Afifi, A. M.; Abass, A. A. Chitosan (PEO)/bioactive Glass Hybrid Nanofibers for Bone Tissue Engineering. RSC Adv. 2014, pp 49144-49152. https://doi.org/10.1039/c4ra06761d.

(21) Thakur, V. K.; Thakur, M. K. Recent Advances in Graft Copolymerization and Applications of Chitosan: A Review. ACS Sustainable Chemistry \& Engineering. 2014, pp 2637-2652. https://doi.org/10.1021/sc500634p.

(22) Ahmed, S.; Annu; Ali, A.; Sheikh, J. A Review on Chitosan Centred Scaffolds and Their Applications in Tissue Engineering. Int. J. Biol. Macromol. 2018, 116, 849-862.

(23) Talebian, S.; Mehrali, M.; Taebnia, N.; Pennisi, C. P.; Kadumudi, F. B.; Foroughi, J.; Hasany, M.; Nikkhah, M.; Akbari, M.; Orive, G.; et al. Self-Healing Hydrogels: The Next Paradigm Shift in Tissue Engineering? Advanced Science. 2019, p 1801664. https://doi.org/10.1002/advs.201801664.

(24) Coutinho, D. F.; Sant, S. V.; Shin, H.; Oliveira, J. T.; Gomes, M. E.; Neves, N. M.; Khademhosseini, A.; Reis, R. L. Modified Gellan Gum Hydrogels with Tunable Physical and Mechanical Properties. Biomaterials 2010, 31 (29), 7494-7502.

(25) Oliveira, A. C. de; de Oliveira, A. C.; Sabino, R. M.; Souza, P. R.; Muniz, E. C.; Popat, K. C.; Kipper, M. J.; Zola, R. S.; Martins, A. F. Chitosan/gellan Gum Ratio Content into Blends Modulates the Scaffolding Capacity of Hydrogels on Bone Mesenchymal Stem Cells. Materials Science and Engineering: C. 2020, p 110258. https://doi.org/10.1016/j.msec.2019.110258.

(26) Coutinho, D. F.; Sant, S.; Shakiba, M.; Wang, B.; Gomes, M. E.; Neves, N. M.; Reis, R. L.; Khademhosseini, A. Microfabricated Photocrosslinkable Polyelectrolyte-Complex of Chitosan and Methacrylated Gellan Gum. J. Mater. Chem. 2012, 22 (33), 17262-17271. 
(27) Yoon, J.; Yang, H.-S.; Lee, B.-S.; Yu, W.-R. Recent Progress in Coaxial Electrospinning: New Parameters, Various Structures, and Wide Applications. Adv. Mater. 2018, 30 (42), e1704765.

(28) Jun, Y.; Kang, E.; Chae, S.; Lee, S.-H. Microfluidic Spinning of Micro- and Nano-Scale Fibers for Tissue Engineering. Lab Chip 2014, 14 (13), 2145-2160.

(29) Waheed, S.; Cabot, J. M.; Macdonald, N. P.; Lewis, T.; Guijt, R. M.; Paull, B.; Breadmore, M. C. 3D Printed Microfluidic Devices: Enablers and Barriers. Lab Chip 2016, 16 (11), 1993-2013.

(30) Ho, C. M. B.; Ng, S. H.; Li, K. H. H.; Yoon, Y.-J. 3D Printed Microfluidics for Biological Applications. Lab Chip 2015, 15 (18), 3627-3637.

(31) Waldbaur, A.; Rapp, H.; Länge, K.; Rapp, B. E. Let There Be Chip-towards Rapid Prototyping of Microfluidic Devices: One-Step Manufacturing Processes. Analytical Methods. 2011, p 2681. https://doi.org/10.1039/c1ay05253e.

(32) Weisgrab, G.; Ovsianikov, A.; Costa, P. F. Functional 3D Printing for Microfluidic Chips. Advanced Materials Technologies. 2019, p 1900275. https://doi.org/10.1002/admt.201900275.

(33) Mi, S.; Du, Z.; Xu, Y.; Sun, W. The Crossing and Integration between Microfluidic Technology and 3D Printing for Organ-on-Chips. J. Mater. Chem. B Mater. Biol. Med. 2018, 6 (39), 6191-6206.

(34) Guzzi, E. A.; Tibbitt, M. W. Additive Manufacturing of Precision Biomaterials. Adv. Mater. 2020, 32 (13), e1901994.

(35) Au, A. K.; Huynh, W.; Horowitz, L. F.; Folch, A. 3D-Printed Microfluidics. Angewandte Chemie International Edition. 2016, pp 3862-3881. https://doi.org/10.1002/anie.201504382.

(36) Li, J.; Baxani, D. K.; Jamieson, W. D.; Xu, W.; Rocha, V. G.; Barrow, D. A.; Castell, O. K. 3D-Printed Microfluidics: Formation of Polarized, Functional Artificial Cells from Compartmentalized Droplet Networks and Nanomaterials, Using One-Step, Dual-Material 3D-Printed Microfluidics (Adv. Sci. 1/2020). Advanced Science. 2020, p 2070005. https://doi.org/10.1002/advs.202070005.

(37) Chan, H. N. The Applications of 3D Printing in Microfluidics and Bioengineering. https://doi.org/10.14711/thesis-b1626108.

(38) Bhattacharjee, N.; Urrios, A.; Kang, S.; Folch, A. The Upcoming 3D-Printing Revolution in Microfluidics. Lab Chip 2016, 16 (10), 1720-1742.

(39) Sun, H.; Jia, Y.; Dong, H.; Dong, D.; Zheng, J. Combining Additive Manufacturing with Microfluidics: An Emerging Method for Developing Novel Organs-on-Chips. Current Opinion in Chemical Engineering. 2020, pp 1-9. https://doi.org/10.1016/j.coche.2019.10.006.

(40) Robinson, T. M.; Hutmacher, D. W.; Dalton, P. D. The Next Frontier in Melt Electrospinning: Taming the Jet. Adv. Funct. Mater. 2019, 29 (44), 1904664.

(41) McMaster, R.; Hoefner, C.; Hrynevich, A.; Blum, C.; Wiesner, M.; Wittmann, K.; Dargaville, T. R.; Bauer-Kreisel, P.; Groll, J.; Dalton, P. D.; et al. Tailored Melt Electrowritten Scaffolds for the Generation of Sheet-Like Tissue Constructs from Multicellular Spheroids. Adv. Healthc. Mater. 2019, 8 (7), e1801326.

(42) Tamayol, A.; Akbari, M.; Annabi, N.; Paul, A.; Khademhosseini, A.; Juncker, D. Fiber-Based Tissue Engineering: Progress, Challenges, and Opportunities. Biotechnology Advances. 2013, pp 669-687. https://doi.org/10.1016/j.biotechadv.2012.11.007.

(43) Leong, M. F.; Toh, J. K. C.; Du, C.; Narayanan, K.; Lu, H. F.; Lim, T. C.; Wan, A. C. A.; Ying, J. Y. Patterned Prevascularised Tissue Constructs by Assembly of Polyelectrolyte Hydrogel Fibres. Nat. Commun. 2013, 4, 2353.

(44) Wan, A. C. A.; Leong, M. F.; Toh, J. K. C.; Zheng, Y.; Ying, J. Y. Multicomponent Fibers by Multi-Interfacial Polyelectrolyte Complexation. Adv. Healthc. Mater. 2012, I (1), 101-105.

(45) Microengineered Multicomponent Hydrogel Fibers: Combining Polyelectrolyte Complexation and Microfluidics. https://doi.org/10.1021/acsbiomaterials.6b00331.s002.

(46) Sant, S.; Coutinho, D. F.; Gaharwar, A. K.; Neves, N. M.; Reis, R. L.; Gomes, M. E.; Khademhosseini, A. Self-Assembled Hydrogel Fiber Bundles from Oppositely Charged Polyelectrolytes Mimic Micro-/Nanoscale Hierarchy of Collagen. Adv. Funct. Mater. 2017, 27 (36), 1606273.

(47) Xu, Z.; Li, Z.; Jiang, S.; Bratlie, K. M. Chemically Modified Gellan Gum Hydrogels with Tunable Properties for Use as Tissue Engineering Scaffolds. ACS Omega 2018, 3 (6), 6998-7007.

(48) Groll, J.; Burdick, J. A.; Cho, D.-W.; Derby, B.; Gelinsky, M.; Heilshorn, S. C.; Jüngst, T.; Malda, J.; Mironov, V. A.; Nakayama, K.; et al. A Definition of Bioinks and Their Distinction from Biomaterial Inks. Biofabrication 2018, 11 (1), 013001 .

(49) Lozano, R.; Stevens, L.; Thompson, B. C.; Gilmore, K. J.; Gorkin, R., 3rd; Stewart, E. M.; in het Panhuis, M.; Romero-Ortega, M.; Wallace, G. G. 3D Printing of Layered Brain-like Structures Using Peptide Modified Gellan Gum Substrates. Biomaterials 2015, 67, 264-273.

(50) Mirabedini, A.; Foroughi, J.; Thompson, B.; Wallace, G. G. Fabrication of Coaxial Wet-Spun Graphene-Chitosan Biofibers. Advanced Engineering Materials. 2016, pp 284-293. https://doi.org/10.1002/adem.201500201.

(51) Martini, B.; Dimida, S.; De Benedetto, E.; Madaghiele, M.; Demitri, C. Study on the Degradation of Chitosan Slurries. Results in Physics 2016, 6, 728-729.

(52) Buriuli, M.; Kumari, W. G.; Verma, D. Evaluation of Hemostatic Effect of Polyelectrolyte Complex-Based Dressings. J. Biomater. Appl. 2017, 32 (5), 638-647.

(53) Nilsen-Nygaard, J.; Strand, S.; Vårum, K.; Draget, K.; Nordgård, C. Chitosan: Gels and Interfacial Properties. Polymers 2015, 7 (3), 552-579.

(54) Rele, S.; Song, Y.; Apkarian, R. P.; Qu, Z.; Conticello, V. P.; Chaikof, E. L. D-Periodic Collagen-Mimetic Microfibers. J. Am. Chem. Soc. 2007, 129 (47), 14780-14787.

(55) Yu, S. M.; Li, Y.; Kim, D. Collagen Mimetic Peptides: Progress Towards Functional Applications. Soft Matter 2011, 7 (18), 7927-7938.

(56) Buriuli, M.; Verma, D. Polyelectrolyte Complexes (PECs) for Biomedical Applications. In Advanced Structured 
Materials; 2017; pp 45-93.

(57) Aguiari, P.; Fiorese, M.; Iop, L.; Gerosa, G.; Bagno, A. Mechanical Testing of Pericardium for Manufacturing Prosthetic Heart Valves. Interact. Cardiovasc. Thorac. Surg. 2016, 22 (1), $72-84$.

(58) Finlay, H. M.; McCullough, L.; Canham, P. B. Three-Dimensional Collagen Organization of Human Brain Arteries at Different Transmural Pressures. J. Vasc. Res. 1995, 32 (5), 301-312.

(59) Everett, J. S.; Sommers, M. S. Skin Viscoelasticity: Physiologic Mechanisms, Measurement Issues, and Application to Nursing Science. Biol. Res. Nurs. 2013, 15 (3), 338-346.

(60) Chattopadhyay, S.; Raines, R. T. Review Collagen-Based Biomaterials for Wound Healing. Biopolymers 2014, 101 (8), 821-833.

(61) Huijing, P. A. Muscle as a Collagen Fiber Reinforced Composite: A Review of Force Transmission in Muscle and Whole Limb. J. Biomech. 1999, 32 (4), 329-345.

(62) Gillies, A. R.; Lieber, R. L. Structure and Function of the Skeletal Muscle Extracellular Matrix. Muscle Nerve 2011. https://doi.org/10.1002/mus.22094.

(63) Amin, K.; Panhuis, M. in H. Mechanical Reinforcement of Wool Fiber through Polyelectrolyte Complexation with Chitosan and Gellan Gum. Fibers Polym. 2013, 1 (3), 47-58.

(64) Malda, J.; Visser, J.; Melchels, F. P.; Jüngst, T.; Hennink, W. E.; Dhert, W. J. A.; Groll, J.; Hutmacher, D. W. 25th Anniversary Article: Engineering Hydrogels for Biofabrication. Adv. Mater. 2013, 25 (36), 5011-5028.

(65) Quigley, A. F.; Razal, J. M.; Thompson, B. C.; Moulton, S. E.; Kita, M.; Kennedy, E. L.; Clark, G. M.; Wallace, G. G.; Kapsa, R. M. I. Nerve Repair: A Conducting-Polymer Platform with Biodegradable Fibers for Stimulation and Guidance of Axonal Growth (adv. Mater. 43/2009). Adv. Mater. 2009, 21 (43). https://doi.org/10.1002/adma.200990160.

(66) Kong, W.; Wang, C.; Jia, C.; Kuang, Y.; Pastel, G.; Chen, C.; Chen, G.; He, S.; Huang, H.; Zhang, J.; et al. Muscle-Inspired Highly Anisotropic, Strong, Ion-Conductive Hydrogels. Adv. Mater. 2018, 30 (39), e1801934. 